\title{
Time machines still over horizon
}

A sixty-year old calculation by Enrico Fermi is discovered to be in error, and inter-atomic signalling between atoms
to be potentially faster than light. But this is not a sign that time machines that defy causality can now be built.

THE idea that the laws of physics are positive interdictions of certain desirable phenomena is much resented. Thus the notion that gravitational forces act indiscriminately, in the direction of the local gravitational field, in proportion only to the mass involved is offensive to those who would take thought and levitate. The common denial that machines capable of doing work (in the technical sense of producing net energy) cannot do so perpetually is often regarded as an intolerable abridgment of the freedom of the human spirit. Not to be able to measure the position and momentum (or any other pair of conjugate variables) simultaneously, as the Uncertainty Principle would have it, is similarly regarded as an affront to the dignity of the human species, even a lie in which malevolent researchers have conspired.

Dr Gerhard C. Hegerfeldt from the University of Göttingen may therefore unwittingly have given a hostage to fortune with his reexamination of the treatment of a problem first tackled in 1932 by Enrico Fermi, at the suggestion of Heisenberg, and now discovered to be flawed (Phys. Rev. Letts $\mathbf{7 2}$, 596-599; 31 January 1994). For Fermi had set out to show that the influence of an excited atom on an (unexcited) neighbour would be communicated no more quickly than the speed of light, and had satisfied the referees of Reviews of Modern Physics (4, $87 ; 1932$ ) that his calculations were correct. If, now, Fermi is discovered to have been mistaken, and the influence is communicated superluminally, then time machines must surely be just around the corner.

In reality, the case of Fermi's excited atom is neither the scandal nor the opportunity it appears, but an instructive illustration of how much has been learned since 1932 . The problem is simply stated. Let there be an atom $A$ excited into its first electronic state and another $B$ at a distance $R$; further suppose that the space between the two is uncomplicated by the presence of radiation. The question is to know the probability that $B$ will be excited by the photon produced by the decay of $A$ from its excited state to the ground state, and in particular to know the least time-delay. Fermi's conclusion was that the time-lag would be at least $R / c$, where $c$ is the velocity of light.

In 1932, the obvious way to calculate the problem was to use Schrödinger's timedependent wave equation to calculate the effect on $B$ of the pulse of electromagnetic energy given off when $A$ decays. The complication of the problem is that the pulse is not instantaneous, but spread out over a (short) length of time related to the linewidth of the corresponding spectral line by the Uncertainty Principle. That means that the pulse of energy sensed by $B$ is not that of a single frequency, but is distributed over all possible frequencies. What seems to have gone wrong in Fermi's treatment is an approximation used to represent a mathematical integral over all positive frequencies. Lesser mortals than Fermi should not take too much pleasure in that.

That something was wrong with Fermi's treatment of this seemingly simple problem appears to have been spotted in the late 1940s. Hegerfeldt gives M. I. Shirokov the credit for telling (in 1962) precisely what the error was, although there have apparently been attempts to save the appearances even in the past few years, presumably incorrectly. Hegerfeldt now reckons to have provided a correct treatment which, among other things, shows that there is a finite if small probability that $B$ will "notice" the decay of $A$ long before the interval $R / c$ has elapsed. Does that not imply that causality in the sense of Einstein's relativity has collapsed, and that the time-machine builders should be licensed to get on with their important work?

That, luckily, is a premature conclusion. Although the formal part of Hegerfeldt's paper is an elegant argument about the representations of operators in an appropriate Hilbert space, which demonstrates yet again that it is possible by elegant mathematics to extract something substantial from nothing, the general interest of his paper is his reformulation of the question Fermi asked himself in 1932. First, the atoms $A$ and $B$ are taken to be strictly independent systems, each with its own system of energy levels unaffected by the other. Second, the intervening vacuum is supposed to be free from photons that may spuriously excite $B$, whether or not $A$ has decayed. But each of these assumptions is, at some level, false. Fermi, in short, had tackled an unphysical problem.

Take the business of the separateness of the two atoms. It is of the essence of quantum mechanics that the electrons in an atom are infinitely spread out physically. The probability of finding one of $A$ 's electrons at some distance may be a rapidly decreasing function of the distance, but it is not strictly zero short of infinity. The assumption that the two atoms are strictly independent is thus strictly false, even though it may be a good starting point from which to embark on a more refined calculation. Accordingly, it should be no surprise that $B$ begins to notice the decay of $A$ before light could have travelled the intervening distance. Of course, the magnitude of the probability that $B$ will respond superluminally does decrease rapidly with the separation distance, but that is not the hole in causality that the timemachine fraternity would concentrate upon.

The assumption that the intervening vacuum is free from photons is even less plausible these days. For one thing, the treatment of the mathematical infinities that arise in the calculation of the properties of electrons in quantum electrodynamics (whimsically, "QED") is crudely the equivalent of surrounding all electrons with a kind of photon cloud, substantially contributing to the overlap between one atom and even a relatively distant neighbour. The "polarization of the vacuum" is the name for this phenomenon, for which Feynman, Schwinger and Tomonaga, with the help of apologists such as Dyson, were responsible late in the 1940s.

QED creates further complications, not surprising when people are forever unearthing evidence that the properties of the electronic states of atoms are affected simply when they are placed in cavities capable of sustaining only some modes and frequencies of electromagnetic radiation. Hegerfeldt puts the philosophical point that, in these circumstances, it may make no sense to say that $B$ can be put into its theoretical ground state, calculated for a strictly isolated atom, when $A$ is somewhere in the neighbourhood, exerting an influence through the intervening vacuum.

Hegerfeldt acknowledges that all three arguments are simply different ways of saying that the two atoms are not strictly separate systems. What his own argument, formal and thus general, does not provide is an estimate of the magnitude of the probability of superluminal signalling between two supposedly independent systems of this kind. It will be interesting to see, when that algebra has been done, whether the superluminal signal is a more rapidly decreasing function of the distance than is the causal signal after an interval of $R / c$. That would be the general expectation.

Whatever the truth, the serious moral in Fermi's error is that approximations used as the basis for calculations, however convenient, may essentially omit some element of the reality they are meant to represent. Meanwhile, those planning to build time machines will have to wait a little longer for the details to emerge.

John Maddox 